\title{
Percutaneous Closure of Patent Foramen Ovale under TEE Guidance in Cryptogenic Stroke and Migraine
}

\author{
Chao Long ${ }^{1}$, Yao Feng ${ }^{1}$, Xiao Lyv ${ }^{1}$, Zheng Lei $^{1}$, Zu Tan ${ }^{1}$, Wen Kuang ${ }^{1}$, and Hao Deng ${ }^{1}$ \\ ${ }^{1}$ Affiliation not available
}

September 25, 2021

\begin{abstract}
Background. To investigate safety and clinical efficacy of percutaneous closure of PFO under TEE guidance in patients with cryptogenic stroke (CS) and migraine, combined PFO and large right-to-left shunt(RLS). Methods. From January 2018 to June 2021 in our hospital, 128 patients were treated by percutaneous PFO occlusion under TEE guidance in CS and migraine. Effective occlusion rate, complications, cerebral ischemia events, migraine relief, and hospitalization costs were analyzed. Postoperative follow-up were conducted. Results. All the 128 patients were successful in percutaneous PFO occlusion. The average operation time was $20^{\sim} 32(25.6 \pm 4.2) \mathrm{min}$, the average hospitalization time was $2^{\sim} 3(2.8 \pm 0.4) \mathrm{d}$. There were 122 patients without RLS and 6 patients with medium residual RLS, with the sealing efficiency of $95.31 \%$ by TTE and right heart contrast echocardiography. 30 patients with migraine underwent percutaneous PFO occlusion, 24 patients relief after operation, with a relief rate of $80.0 \%$. Postoperative follow-up was conducted for 1-36(28.8+-3.6)months, and no follow-up was lost. 98 cases of CS with PFO underwent percutaneous blockade were followed up without cerebral embolism recurrence, 26 of 30 patients with migraine received significant remission of migraine, with remission rate of $86.66 \%$. There were no serious adverse events. Conclusions. For patients with CS and migraine, combined PFO and large RLS, percutaneous PFO occlusion under the guidance of TEE has fewer complications and is safe and effective. It can prevent the recurrence of CS and treat migraine.
\end{abstract}

Percutaneous Closure of Patent Foramen Ovale under TEE Guidance in Cryptogenic Stroke and Migraine Chaozhong Long, Yaoguang Feng ${ }^{*}$, Xiaolin Lyv, Zhengwen Lei,

Zusong Tan, Wenan Kuang, and Hao Deng

The First Affiliated Hospital, Department of Cardiovascular Surgery, Hengyang Medical School, University of South China, Hengyang, Hunan, 421001, China

* Corresponding author: Feng Yao-guang, Email:fengyaog@hotmail.com

Abstract:

Background . To investigate safety and clinical efficacy of percutaneous closure of PFO under TEE guidance in patients with cryptogenic stroke (CS) and migraine, combined PFO and large right-to-left shunt(RLS).

Methods . From January 2018 to June 2021 in our hospital, 128 patients were treated by percutaneous PFO occlusion under TEE guidance in CS and migraine. Effective occlusion rate, complications, cerebral ischemia events, migraine relief, and hospitalization costs were analyzed. Postoperative follow-up were conducted.

Results. All the 128 patients were successful in percutaneous PFO occlusion. The average operation time was $20^{\sim} 32(25.6 \pm 4.2) \mathrm{min}$, the average hospitalization time was $2^{\sim} 3(2.8 \pm 0.4)$ d. There were 122 patients without RLS and 6 patients with medium residual RLS, with the sealing efficiency of $95.31 \%$ by TTE and right heart contrast echocardiography. 30 patients with migraine underwent percutaneous PFO occlusion, 
24 patients relief after operation, with a relief rate of $80.0 \%$. Postoperative follow-up was conducted for 136(28.8+-3.6)months, and no follow-up was lost. 98 cases of CS with PFO underwent percutaneous blockade were followed up without cerebral embolism recurrence, 26 of 30 patients with migraine received significant remission of migraine, with remission rate of $86.66 \%$. There were no serious adverse events.

Conclusions. For patients with CS and migraine, combined PFO and large RLS, percutaneous PFO occlusion under the guidance of TEE has fewer complications and is safe and effective. It can prevent the recurrence of CS and treat migraine..

\section{KEYWORDS}

transesophageal echocardiography, patent foramen ovale, cryptogenic stroke,

migraine, percutaneous closure

\section{Introduction}

Patent foramen ovale (PFO) is a common abnormality, occurring in $20-34 \%$ of the population. Some PFO can open widely, enabling a paradoxical embolus to transit from the venous to arterial circulation, which is associated with stroke and systemic embolisation [1]. It has been implicated in a number of pathologies, such as cryptogenic stroke (CS), platypnea orthodeoxia syndrome, decompression sickness, and migraine with auras [2]. Recent trials have shown that closure of PFO, especially if associated with an atrial septal aneurysm and/or a large interatrial shunt, may reduce the risk of recurrent stroke as compared to medical treatment $[3,4]$. Percutaneous closure is conventionally performed under the guidance of fluoroscopy. Nevertheless, radiation exposure during fluoroscopy represents a risk to the patient and medical staff. Therefore the development of a safe and less invasive procedure with less radiation exposure is important, and transesophageal echocardiography (TEE) was generally used in patients with clear sound window, transcatheter ASD closure guided by TEE is proposed [5,6]. Our hospital has carried out TEE-guided percutaneous PFO closure without fluoroscopy since 2018, we retrospectively reviewed cases to evaluate the feasibility, safety, and effectiveness of this approach.

\section{Methods}

2.1. Patients. Patients were diagnosed with CS or migraine with PFO in our hospital from January 2018 to June 2021. There were 128 patients who received TEE-guided percutaneous PFO closure in our department, including 98 patients with CS, 30 patients with migraine, combined PFO and large right-to-left shunt (RLS). There were 52 male patients and 76 female patients. The patients' mean age was from 26 to 55 (42.60+-8.50) years. Valsalva action PFO (5.8+-1.2) mm. All patients with brain MRI examination, transthoracic echocardiography (TTE), contrast-enhanced transcranial doppler (cTCD), and transesophageal echocardiography (TEE). This study was approved by institutional review boards of our hospital, all patients signed the statement of informed consent.

2.2. Ultrasonic evaluation. Preoperative routine TEE examination in all patients. The morphology, size, tunnel length, and structural characteristics of PFO were observed in multiple sections, including whether there was atrial septal dilatoma, resting RLS, or large amount of RLS, combined with inferior vena cava valve $>10 \mathrm{~mm}$ or Chiari's network and other PFO anatomical risk factors, except other cardiac factors causing cerebral apoplexy. RLS was graded by contrast-transthoracic echocardiography (cTTE) in terms of the number of microbubbles present in the left ventricular cavity on a single frame of still images. CTCD adopts the two-sided criterion of microbubble number grading, which can be used to know whether there is RLS or not and its shunt size in detail.

2.3. Procedure. Tracheal intubation under general anesthesia, PFO was reevaluated from different perspectives by TEE, right femoral vein was punctured. After intravenous heparin injection (100 units $/ \mathrm{kg}$ ), the $5 \mathrm{~F}$ single-curved catheter was sent to the middle of the left atrium through PFO under the guidance of TEE, and a $260 \mathrm{~mm}$ long and hard guidewire was sent to the left upper pulmonary vein or left atrial appendage along the catheter. Withdraw the $5 \mathrm{~F}$ single-bend catheter and send the long sheath tube along the guide wire 
to the middle of the left atrium. The guide was inserted into the delivery sheath, and the occlusion device was fed along the delivery sheath into the left atrium. The left atrial plate was released after withdrawal of the delivery system to close the atrial septum and the outer sheath was retracted to release the occlusion device waist and right atrial plate, so that the atrial septum was between the left and right umbrella folder. Multiple TEE view and push- pull tests were used to assess the location of the occluding device, the presence of residual shunt, the condition of the valve, the relationship of the occluding device to the coronary sinus, superior vena cava, inferior vena cava, and pulmonary vein, and the presence or absence of pericardial effusion. After TEE confirmed that the position and shape of the sealing device were good, the sealing device was released and the pressure was applied to stop bleeding after surgery. Domestic PFO occlusion device (produced by Beijing Starway Medical Technology Inc) or imported Amplatzer PFO occlusion device was selected, and domestic ASD occlusion device (produced by Shanghai Shape Memory Alloy Material Co. Ltd) was selected for some patients with atrial septal dilatoma (Figure1).

Figure1. The procedure of percutaneous closure of PFO under the guidance of TEE.

A. The morphology, size, tunnel length, and structural characteristics of PFO were evaluated in multiple sections. B. The catheter and sheath were withdrawn while maintaining the guide wire in the left atrium. C. A $9 \mathrm{Fr}$ to $10 \mathrm{Fr}$ delivery sheath was then selected according to the diameter of the occlude and was inserted into the left atrium along the guide wire. D. PFO occluder was then inserted for percutaneous closure of PFO under TEE guidance.

2.4. Follow-up. TTE and electrocardiography were performed in both groups at 1 month, 3 months, 6 months, and 1 year after discharge and annually thereafter. Patients were followed up by TTE to assess cardiac function, occlusion device morphology, residual shunt status, and other indicators. Additionally, the patients' chest X-rays and electrocardiograms were double-checked in cases involving complications. cTCD examination was also performed at 1 and 3 months after PFO plugging to detect whether there was residual medium or large amount of RLS shunt and evaluate the blocking effect. At the same time, the success rate, effective occlusion rate, complications (pericardial effusion, occluder displacement, peripheral hematoma, etc.), recurrence of ischemic stroke, relief of migraine, and other adverse events, including bleeding and new arrhythmia, were observed with follow-up

2.5. Statistical Analysis. Descriptive statistics were used for patients' characteristics. Continuous variables with normal distribution are presented as mean+-standard deviation. All statistical analyses were performed using the SPSS software (version 24.0 for Windows).

\section{Results}

3.1. Operation situation. All the 128 patients with TEE-guided percutaneous PFO closure were successful, and one of them showed poor stability of the closure umbrella, and the replacement of a larger closure umbrella was successful. The average operation time (from puncture to removal of sheath tube) of the patients was $20^{\sim} 32(25.6+-4.2) \mathrm{min}$, the average hospitalization time was $2^{\sim} 3(2.8+-0.4) \mathrm{d}$, and the hospitalization cost was 2.8-3.2 (3.0+-0.5) ten thousand RMB. After the operation, cTCD examination showed that there were 122 patients without large number of RLS shunts, and the effective rate was $95.31 \%$. In the residual RLS shunt, there were 6 cases, but the position of the sealer was fixed and there was no shift. 30 patients with migraine underwent percutaneous PFO occlusion, 24 patients relief after operation, with a relief rate of $80 \%$. All the patients recovered and were discharged from the hospital without peripheral vascular injury or pericardial effusion and other complications after surgery. The clinical effect of percutaneous PFO under the guidance of TEE were shown in (Table 1).

3.2. Follow-up. Postoperative follow-up was conducted from 1 to 36(28.8+-3.6) months, and no follow-up was lost. CTCD was added to determine whether there was residual shunt of RLS at 1 and 3 months of follow-up. TTE, chest X-ray, and electrocardiogram were reexamined at other times, and brain MRI was performed if necessary. 98 cases of CS with PFO underwent percutaneous blockade were followed up without cerebral embolism recurrence. 30 cases of PFO occluder treated patients had significantly relieved migraine in 26 cases, with a remission rate of $86.7 \%$ after 3 months. There was no significant remission in 4 migraine 
patients, but the PFO occluder was stable, and cTCD examination showed no medium-large amount of RLS. After 3 months, due to the intima coverage on the surface of the sealing device, only 4 case was followed up to observe a small-medium amount of RLS shunt in the residual, and the position of the sealing device was fixed without displacement or thrombosis. No patients suffered from stroke recurrence, migraine recurrence, pericardial effusion, occluder displacement, new atrial fibrillation, residual shunt, thrombosis and new valve regurgitation complications occurred.

\section{Discussion}

4.1. PFO closure prevent recurrent stroke. The effect of PFO closure on stroke recurrence was greatest in patients with an ASA or large shunt (RR 0.27, 95\% CI: 0.11-0.70, P=0.01) [3]. Several case-control studies have shown that PFO prevalence was three times higher in patients with cryptogenic stroke than in controls without stroke or stroke patients with a known cause, and this difference was amplified to five times higher in younger adults (under 55 years of age) with cryptogenic stroke. The association between PFO and cryptogenic stroke is stronger if there is an ASA $[2,7]$. Younger patients with high RoPE scores will benefit the most from PFO closure[8]. In this study review cTCD 6 cases exist medium to large of RLS, 122 cases had no shunt or a small amount of RLS, and the effective success rate of plugging was $95.31 \%$ after surgery. With the endothelialization of the occluder, residual shunt will subsequently improved, postoperative 3 months review cTCD when only 2 patient still exist small-medium of RLS, postoperative march plugging effectively the success rate of $98.4 \%$, significantly higher than the literature reported[3,9], it has to do with this research strictly grasp the indications made plugging, the guidance of TEE is more clear, at the same time for long tunnel or merge septal dilatoma patients chosen ASD closure, the blocking effect is more accurate.

4.2. TEE-guided percutaneous PFO closure. The most significant feature of this technique is to use echocardiography as the only guidance tool for percutaneous interventional therapy, and to abandon the previous X-ray guidance method to avoid the exposure of the surgeon and patients, to radiation and the injury of X-rays and contrast agents[5,6]. TEE is considered as the gold standard for PFO detection, and the sensitivity and specificity are $100 \%$. Echocardiography can visually display the impact of the occluder on the mitral valve, coronary sinus, and pulmonary vein, and real-time monitoring of the occluder release process, which allows for occlude replacement if it is found to be incorrectly positioned $[2,5,6]$. This study analyzed 128 patients in our hospital who were successful in the percutaneous PFO closure surgery under the guidance of TEE, and one patient showed poor stability of the occluder. The main reason was that PFO was long tunnel type and the primary compartment was weak, which was then recovered and replaced with a larger PFO occluder. Postoperative follow-up of 1-36(28.8+-3.6)months, 128 cases were made of postoperative follow-up, not yet pericardial effusion, occluder shift and erosion, atrioventricular block, new atrial fibrillation, residual shunt, thrombosis and new complications such as valvular regurgitation. The results show that TEE-guided percutaneous PFO blocking operation is safe and effective, this technique avoids the radiation and use of contrast agents in selected patients, and the near and medium efficacy of CS recurrence prevention is satisfactory.

4.3. Migraine and PFO. Multiple studies have reported a significant association between migraine headaches and the presence of PFO, particularly migraine with aura. A 2016 meta-analysis pooled 21 case-control studies ( $\mathrm{n}=5,572)$ to show that there is higher prevalence of migraine (OR 2.46, 95\% CI: $1.55-3.91, \mathrm{P}=0.0001$ ) and migraine with aura (OR 3.36, 95\% CI: 2.04-5.55, $\mathrm{P}<0.001$ ) in $\mathrm{PFO}$ patients compared to non-PFO patients [10]. Studies have also demonstrated a right-to-left shunt in $41-48 \%$ of patients with migraine with aura [11]. In this study, 30 migraine patients with PFO and medium-large RLS shunt were blocked by PFO, and 24 migraine patients were relieved after surgery, with a remission rate of $80 \%$. Three months after the surgery, 30 patients with PFO blocking therapy were reexamined, and 26 of them experienced significant relief of migraine, with a remission rate of $86.7 \%$, which was consistent with literature reports[10,11]. 4 migraine patients showed no significant remission, but the PFO occluder was stable, and cTCD examination showed no medium - large amount of RLS, indicating that there were many reasons for migraine, and medium - large amount of RLS in PFO combination may only be one of the main reasons.

4.4. Advantages and indications of percutaneous PFO closure. The cumulative Kaplan-Meier 5-year estimate 
of the probability of stroke was $4.9 \%$ in the antiplatelet group [12]. The results from the Gore-REDUCE trial therefore supported the findings of other trials that transcatheter PFO closure was superior to medical therapy alone in patients with cryptogenic stroke, with an NNT $=28$ to prevent one stroke in 24 months. Importantly, this trial carefully selected patients who were more likely to have stroke attributable to PFO, hence increasing the likelihood that PFO closure would be effective $[3,9,13,14]$. In brief, these studies indicate that in patients aged $<60$ years, PFO closure probably confers a reduction in ischemic stroke recurrence compared with antiplatelet therapy alone, while it also incurs a risk of persistent atrial fibrillation and device-related adverse events $[14,15]$. Patients with presumably pathogenic PFOs who are likely to benefit most from closure over medical therapy should be identified, and specific features that suggest the greatest benefit are the presence of an ASA or a large right-to-left shunt. The results of PFO were consistent with those of this group. Postoperative follow-up of 1-36(28.8+-3.6)months showed no new atrial fibrillation, which was considered to be related to strict case selection, small sample size, short follow-up time, and appropriate selection of TEE-guided sealer size.

\section{Conclusions}

This study showed that for CS and migraine with PFO and large RLS patients, TEE-guided percutaneous PFO closure had fewer complications, and the surgery was safe and effective. It can prevent CS recurrence and relieve migraine. It is essential to choose surgical indications carefully, standardize operation procedure, and strengthen long-term follow-up.

\section{Conflicts of Interest}

The authors have no conflicts of interest to declare.

\section{Funding Statement}

Project Supported by the Natural Science Foundation of Hunan Province, China (Grant No. 2020JJ4546);

\section{References}

[1] Calvert PA, Rana BS, Kydd AC, et al. Patent foramen ovale: anatomy, outcomes, and closure. Nat Rev Cardiol. 2011, 8(3):148-60.

[2] Mojadidi MK, Zaman MO, Elgendy IY, et al. Cryptogenic Stroke and Patent Foramen Ovale. J Am Coll Cardiol. 2018; 71 (9):1035-1043.

[3] Mohammad Abdelghani, Sahar A O El-Shedoudy, Martina Nassif , et al. Management of Patients with Patent Foramen Ovale and Cryptogenic Stroke: An Update. Cardiology. 2019; 143(1):62-72.

[4] Vaidya K, Khandkar C, Celermajer D.Current management aspects in adult congenital heart disease: non-surgical closure of patent foramen ovale. Cardiovasc Diagn Ther. 2018; 8(6):739-753.

[5] Xu WZ, Shou XY, Li JH, et al. Non-fluoroscopic percutaneous transcatheter closure of atrial septal defects in children under transesophageal echocardiographic guidance. World J Pediatr. 2018; 14(4):378-382.

[6] Pan XB, Ou-Yang WB, Pang KJ, et al. Percutaneous closure of atrial septal defects under transthoracic echocardiography guidance without fluoroscopy or intubation in children. J Interv Cardiol. 2015; 28(4):3905 .

[7] Lattanzi S, Brigo F, Cagnetti C, et al. Patent Foramen Ovale and Cryptogenic Stroke or Transient Ischemic Attack: To Close or Not to Close? A Systematic Review and Meta-Analysis. Cerebrovasc Dis. 2018; 45(5-6):193-203.

[8] Bedeir K, Volpi J, Ramlawi B. Cryptogenic Stroke with a Patent Foramen Ovale: Medical Therapy, Percutaneous Intervention, or Surgery. J Card Surg. 2016; 31(3):156-60.

[9] Diener HC, Gerloff C, Thaler DE, et al. Closure of patent foramen ovale and cryptogenic stroke: unresolved issues. Curr Neurol Neurosci Rep. 2018;18(12):92. 
[10] Takagi H, Umemoto T. A meta-analysis of case-control studies of the association of migraine and patent foramen ovale. J Cardiol. 2016 ; 67(6): 493-503.

[11] Tobis JM, Charles A, Silberstein SD, et al. Percutaneous Closure of Patent Foramen Ovale in Patients With Migraine: The PREMIUM Trial. J Am Coll Cardiol. 2017; 70(22): 2766-2774.

[12] Mas JL, Derumeaux G, Guillon B, et al. Patent foramen ovale closure or anticoagulation vs. antiplatelets after stroke. N Engl J Med. 2017; 377(11):1011-1021.

[13] Sondergaard L, Krasner SE, Rhodes JF, et al. Patent foramen ovale closure or antiplatelet therapy for cryptogenic stroke. N Engl J Med. 2017Sep, 377(11), 1033-1042.

[14] Kasner SE, Swaminathan B, Lavados P, Sharma M, Muir K, Veltkamp R,et al. Rivaroxaban or aspirin for patent foramen ovale and embolic stroke of undetermined source: a prespecified subgroup analysis from the NAVIGATE ESUS trial. Lancet Neurol .2018; 17(12):1053-1060.

[15] Stortecky S, da Costa BR, Mattle HP, et al. Percutaneous closure of patent foramen ovale in patients with cryptogenic embolism: a network meta-analysis. Eur Heart J. 2015; 36(2): 120-128.

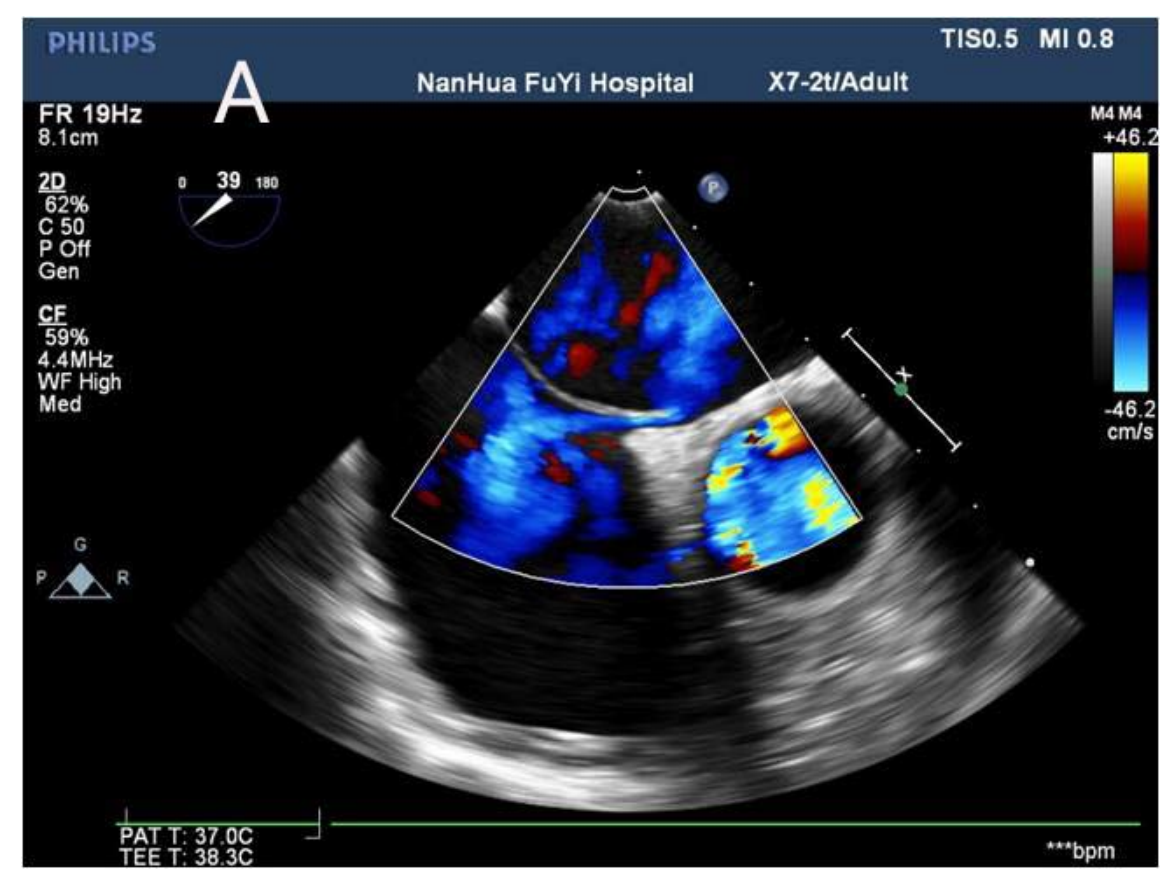



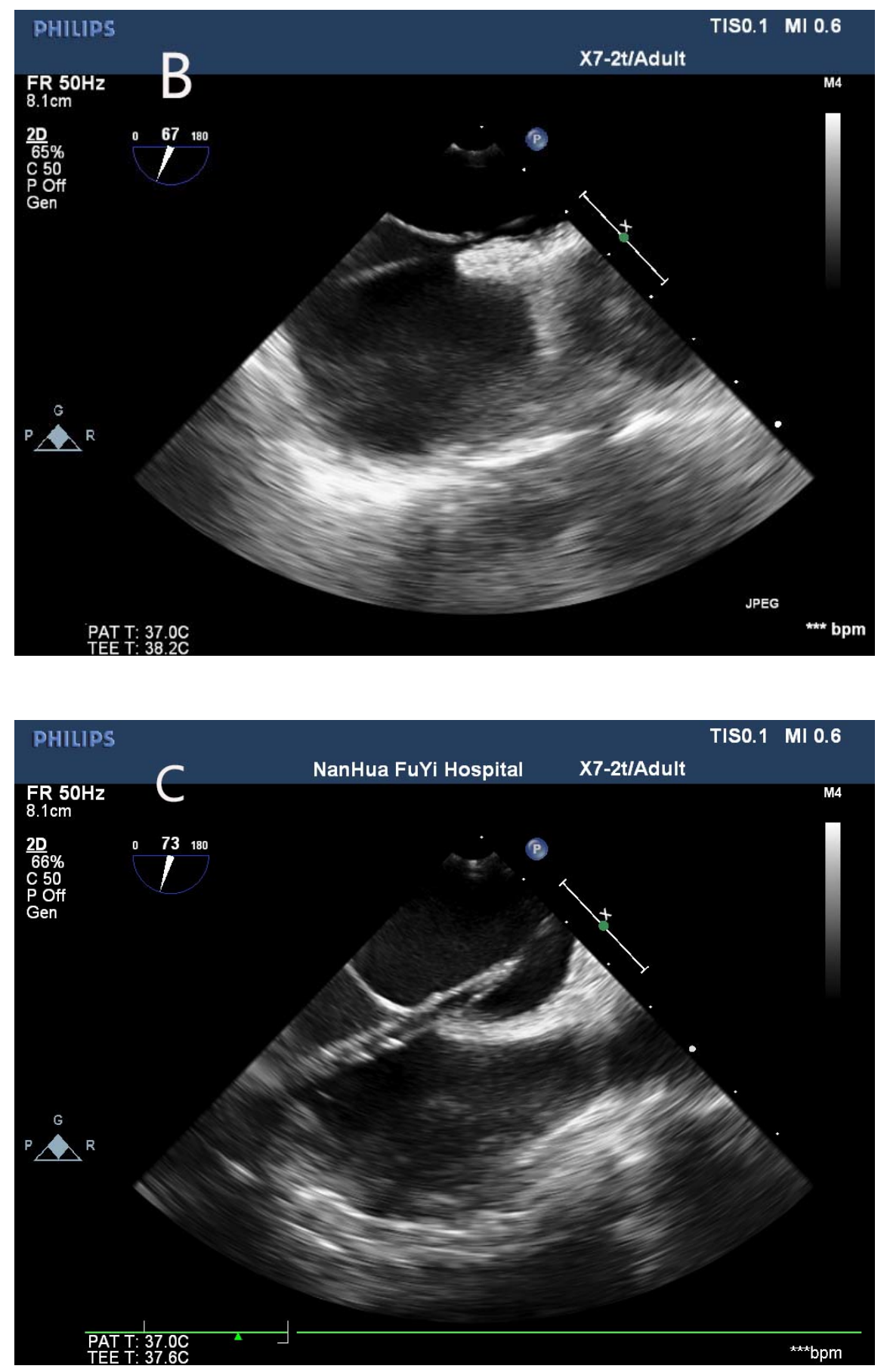


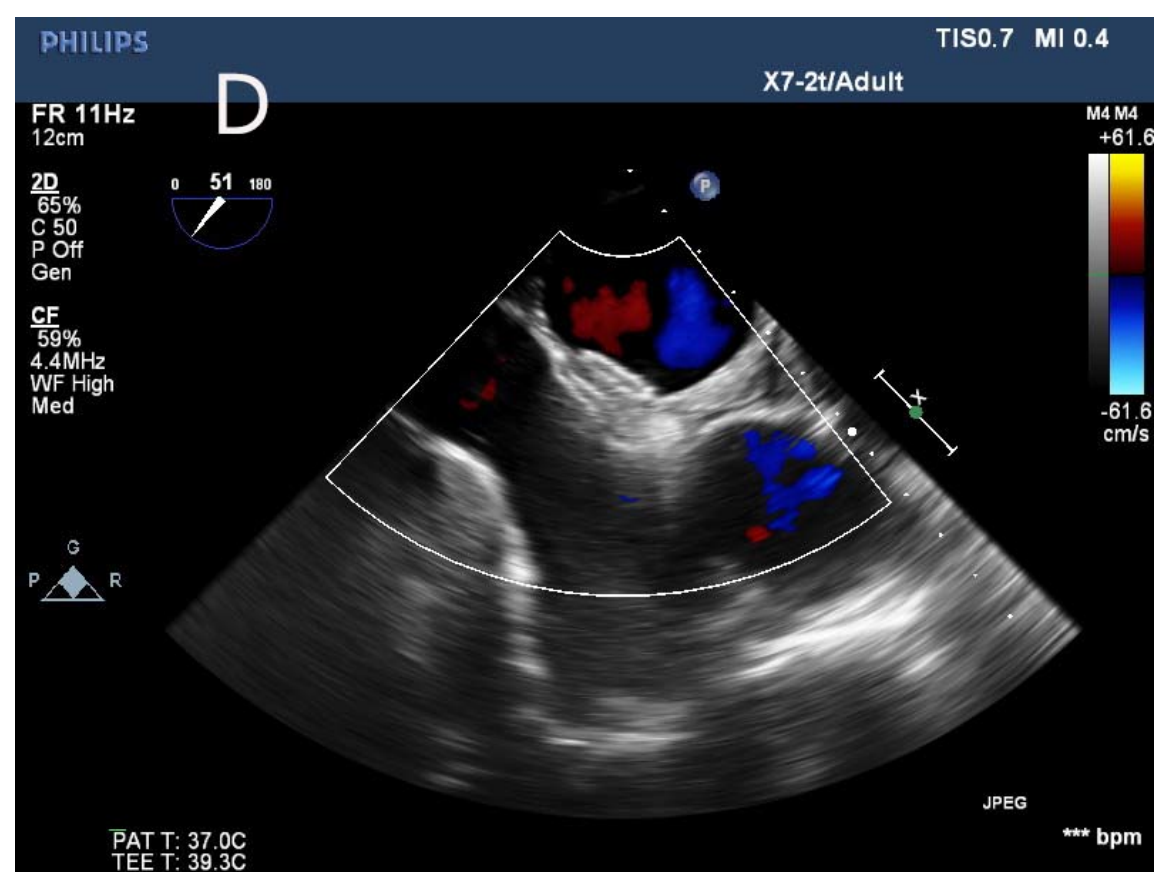

Hosted file

Table 1.docx available at https://authorea.com/users/437816/articles/539189-percutaneousclosure-of-patent-foramen-ovale-under-tee-guidance-in-cryptogenic-stroke-and-migraine 